\title{
Not fade away: the HPA axis and depression
}

\author{
P. J. Cowen* \\ University Department of Psychiatry, Warneford Hospital, Oxford, UK
}

\begin{abstract}
Salivary cortisol sampling has confirmed the presence of increased cortisol secretion in depression and has also revealed that some aspects of hypothalamo-pituitary-adrenal (HPA) axis disturbance have trait-like characteristics that may predict the risk of future illness. Persistent hypersecretion of cortisol in individuals vulnerable to depression also has implications for the medical co-morbidities associated with mood disorder. Pharmacological treatments targeted at the HPA axis represent a novel approach to the management of depression and its complications; however, a better understanding of the molecular basis of HPA axis dysfunction in depressed patients will be needed before this promise can be fulfilled.
\end{abstract}

Received 16 January 2009; Revised 11 February 2009; Accepted 11 February 2009; First published online 1 April 2009

Key words: Cortisol, depression, glucocorticoid receptor, HPA axis.

\section{Introduction}

The persistence of antediluvian pathophysiological theories in psychiatry can be attributed to a number of factors; for example, the absence of anything better to replace them or the pernicious influence of drug company marketing strategies (Cowen, 2008). However, the link between cortisol hypersecretion and depression is based on repeated clinical observations, which have easily outlived attempts, including my own, to debunk them (Mullen et al. 1986; Cowen, 2002). The problem is rather to know what the phenomenon of excessive cortisol secretion in depression represents and whether it can be used for predictive or therapeutic purposes. Here I summarize recent relevant findings and outline a few of the many questions prompted by these observations.

\section{Use of salivary cortisol to assess hypothalamo-pituitary-adrenal (HPA) axis activity}

The field has been transformed by the use of salivary cortisol measurement, which enables the sampling of large numbers of participants in ecologically valid conditions (Pruessner et al. 1997). The stimulus of waking provides an 'endogenous' challenge to the HPA axis; this enables a dynamic measure of HPA activity, which is time-locked to the point of waking, thereby removing an important source of variation in 'baseline' measures of early morning cortisol secretion.

* Address for correspondence: Professor P. J. Cowen,

Neurosciences Building, Warneford Hospital, Oxford OX3 7JX, UK.

(Email: phil.cowen@psych.ox.ac.uk)
It seems that the salivary cortisol response to waking is more sensitive to moderate degrees of depression than traditional measures such as the dexamethasone suppression test. For example, failure to suppress to dexamethasone is associated with more severe depressive states such as melancholia and in-patient status. Rates of dexamethasone non-suppression in moderately depressed out-patients are low (Nelson \& Davis, 1997). In such subjects, however, there is a clear elevation of waking salivary cortisol (Bhagwagar et al. 2005). It will be important to determine whether the apparent increase in sensitivity of the waking cortisol measure to detect depression is at the cost of lowered sensitivity. For example, are waking salivary cortisol levels increased in people with anxiety or adjustment disorders?

\section{Trait or state?}

Previous studies with the dexamethasone suppression test suggested that abnormal HPA axis activity was related to the presence of an acute depressive state, in that clinical recovery was associated with a return to normal suppressor status, and in patients where this did not occur, the risk of relapse was high (Ribeiro et al. 1993). However, the increase in waking salivary cortisol found in acutely depressed patients seems to have trait-like characteristics. For example, secretion is elevated in patients recovered from depression and is also abnormally high in people with no personal history of the disorder but who are at increased risk because of high neuroticism scores or a parental history of depression (Bhagwagar et al. 2003; Portella et al. 2005; Mannie et al. 2007). 
These findings suggest that, although some aspects of HPA axis abnormality resolve with improvement in depressive symptoms, others are more persistent and in fact may be present before the onset of clinical illness, thereby representing a vulnerability marker (Bhagwagar \& Cowen, 2008). Indeed, there is evidence to suggest that elevated salivary cortisol secretion is predictive of future depressive episodes in both young people and adults (Goodyer et al. 2000; Harris et al. 2000). From this viewpoint elevated salivary cortisol secretion has some of the characteristics of an endophenotype of depression and could be a suitable marker for genetic analysis. Twin studies have revealed that waking salivary cortisol secretion does show a substantial heritability (Wust et al. 2000) but the contribution of genetic polymorphisms in specific genes such as those encoding the corticosteroid receptors is not well established. It is possible that carriers of the short allele of the serotonin transporter have elevated waking cortisol levels but the data are not consistent (Mannie et al. 2008).

Although abnormalities in the HPA axis may be partly genetic, it is also possible that they stem from early adverse experiences. For example, early separation of non-human primates from their mothers can lead to abnormalities in the regulation of cortisol secretion that persist into adult life (Pariante \& Lightman, 2008). The children of mothers with postnatal depression show cortisol hypersecretion and although genetic factors could play a role, it is equally possible that disturbances in early attachment associated with the presence of maternal depressive symptoms could lead to abnormal HPA axis development (Halligan et al. 2004).

\section{Cortisol and the brain}

If cortisol hypersecretion predisposes to depression, what might be the mechanism? Corticosteroids may have a direct effect on mood. For example, treatment with pharmacological doses of steroids can cause clinically significant mood changes and the cortisol hypersecretion of Cushing's disease is often associated with depression, which resolves when plasma cortisol levels are lowered. Such observations have led to the development of cortisol synthesis inhibitors and glucocorticoid antagonist strategies for the treatment of acute depression.

In most patients, however, it seems unlikely that depressed mood is a direct consequence of increased cortisol secretion. For example, as noted earlier, waking cortisol secretion is elevated in people with a history of depression, independent of current mood state. However, there is increasing knowledge of the neurobiological consequences of excessive cortisol secretion and there are several ways in which these consequences could be linked to increased risk of depression and associated morbidities.

A particularly interesting recent discovery is that the brain retains the ability to manufacture new neurons in regions such as the hippocampus throughout life. This neurogenesis is inhibited by excessive cortisol secretion. In animal experimental studies there is also evidence that excess cortisol secretion can facilitate neurotoxicity, again particularly in the hippocampus. Structural imaging has revealed that patients with recurrent depression exhibit evidence of hippocampal atrophy, which could be a long-term consequence of excess cortisol secretion (see Herbert et al. 2006).

These effects of cortisol can be linked to cognitive impairment, particularly in declarative memory. Such deficits are commonly recognized in acute depression but they can also persist between episodes, and a similar deficit has been identified in non-depressed young people who hypersecrete cortisol (Mannie et al. 2008). The abnormalities in the latter group are subtle and certainly not clinically detectable; however, it is conceivable that they may in some way contribute to difficulties, for example in problem solving, and could thereby increase the risk of affected individuals encountering adverse environments. Although it is known that cortisol facilitates emotional memory, there have been few studies of its effects on other aspects of emotional processing; this is likely to be a fruitful area for future research.

\section{Cortisol and general medical conditions}

Recurrent or chronic depression is associated with increased risk of several general medical conditions including obesity, diabetes, hypertension, ischaemic heart disease and osteoporosis. There are likely to be several mechanisms involved, such as the effects of depression to decrease self-care, and also the liability of some antidepressant medications to promote weight gain. However, it is also possible that increased cortisol secretion could underpin some of these adverse medical outcomes. For example, patients with Cushing's disease frequently experience obesity, hypertension and diabetes (see Brown et al. 2004; Farmer et al. 2008).

The fact that patients who have recovered from depression continue to hypersecrete cortisol means that the risk of adverse health consequences may continue to develop even in individuals in symptomatic remission. The same could conceivably apply to people who hypersecrete cortisol through a family history of depression but who have not themselves experienced depressive illness. If this is the case it has important 
implications for the identification of people at high risk of a range of highly morbid general medical conditions and perhaps eventually for their prevention through pharmacological or behavioural strategies designed to lower cortisol secretion.

\section{Advances in treatment}

There have been great hopes that pharmacological manipulation of the HPA axis might provide a novel means of treating depression. Thus far, these hopes have not been realized, although there are hints that antagonists at the corticotrophin-releasing hormone $(\mathrm{CRH})$ receptor may have therapeutic potential. There are as yet no convincing data that glucocorticoid receptor (GR) antagonists have generally useful antidepressant activity although there are data suggesting positive effects on cognitive function in bipolar disorder (Pariante \& Lightman, 2008).

One problem in the design of pharmacological strategies aimed at the HPA axis is an incoherence in the theories linking cortisol hypersecretion and depression. For example, GR receptor antagonists might block the cellular effects of excessive cortisol in depressed patients, which is why these agents were developed as antidepressants. However, treatment of depressed patients with such drugs leads to greatly elevated plasma cortisol levels that might, for example, increase activity at mineralocorticoid receptors. At the same time some have argued that cortisol hypersecretion in depression is in fact due to impaired GR receptor function, which leads to diminished efficacy of cortisol feedback control. If this is the case, GR receptor antagonism might not be a helpful strategy. The development of new drug treatments probably needs a more detailed understanding of the specific molecular mechanisms that underlie cortisol hypersecretion in depressed patients (Pariante, 2006).

\section{Conclusions}

Discussions of the role of the HPA axis in depression inevitably throw up clinically relevant questions that attest to the vibrancy of this research area. Developments in neuroscience have increased the tractability of many of the questions that face us. Indeed, one of the fascinations of the field is the many levels on which the role of cortisol in depression may be played out and investigated, from effects on neurogenesis through changes in learning, memory and emotional processing to clinical trials of novel agents. The development of pharmacological tools to modify HPA axis activity should eventually lead to new avenues of therapy probably with indications rather wider than the acute treatment of major depression. A long road probably remains, but even psychiatrists can be encouraged by Einstein's comment, 'It's not that I'm so smart, it's just that I stay with problems longer.'

\section{Acknowledgements}

The studies of the author have been supported by the Medical Research Council (MRC).

\section{Declaration of Interest}

P.J.C. has served as paid member of advisory boards for the following companies developing antidepressant medications: DSM, Lundbeck, Eli Lilly, Servier, Wyeth, Xytis.

\section{References}

Bhagwagar Z, Cowen PJ (2008). 'It's not over when it's over' : persistent neurobiological abnormalities in recovered depressed patients. Psychological Medicine 38, 307-313.

Bhagwagar Z, Hafizi S, Cowen PJ (2003). Increase in concentration of waking salivary cortisol in recovered patients with depression. American Journal of Psychiatry 160, 1890-1891.

Bhagwagar Z, Hafizi S, Cowen PJ (2005). Increased salivary cortisol after waking in depression. Psychopharmacology 182, 54-57.

Brown ES, Varghese FP, McEwen BS (2004). Association of depression with medical illness: does cortisol play a role. Biological Psychiatry 55, 1-9.

Cowen PJ (2002). Cortisol, serotonin and depression: all stressed out? British Journal of Psychiatry 180, 99-100.

Cowen PJ (2008). Serotonin and depression: pathophysiological mechanism or marketing myth? Trends in Pharmacological Sciences 29, 433-436.

Farmer A, Korszun A, Owen MJ, Craddock N, Jones L, Jones I, Gray J, Williamson RJ, McGuffin P (2008). Medical disorders in people with recurrent depression. British Journal of Psychiatry 192, 351-355.

Goodyer IM, Herbert J, Tamplin A, Altham P (2000). Recent life events, cortisol, dehydroepiandrosterone and the onset of major depression in high risk adolescents. British Journal of Psychiatry 177, 499-504.

Halligan SL, Herbert J, Goodyer IM, Murray L (2004). Exposure to postnatal depression predicts elevated cortisol in adolescent offspring. Biological Psychiatry 55, 171-176.

Harris T, Borsanyi S, Messari S, Stanford K, Cleary S, Shiers H, Brown GJH (2000). Morning cortisol as a risk factor for subsequent major depressive disorder in adult women. British Journal of Psychiatry 177, 505-510.

Herbert J, Goodyer IM, Grossman AB, Hastings $\mathrm{MH}$, de Kloet ER, Lightman SL, Lupien SJ, Roozendaal B, Seckl JR (2006). Do corticosteroids damage the brain? Journal of Neuroendocrinology 18, 393-411.

Mannie ZN, Barnes J, Bristow GC, Harmer CJ, Cowen PJ (2008). Memory impairment in young women at increased risk of depression: influence of cortisol and 5-HTT 
genotype. Psychological Medicine. Published online 8 September 2008. doi:10.1017/S0033291708004248.

Mannie ZN, Harmer CJ, Cowen PJ (2007). Increased waking salivary cortisol levels in young people at familial risk of depression American Journal of Psychiatry 164, 617-621.

Mullen PE, Linsell CR, Parker D (1986). Influence of sleep disruption and calorie restriction on biological markers for depression. Lancet 2, 1051-1055.

Nelson JC, Davis JM (1997). DST studies in psychotic depression: a meta-analysis. American Journal of Psychiatry 154, 1497-1503.

Pariante CP (2006). The glucocorticoid receptor: part of the solution or part of the problem? Journal of Psychopharmacology 20, 79-84.

Pariante CM, Lightman SL (2008). The HPA axis in major depression: classical theories and new developments. Trends in Neurosciences 31, 464-468.
Portella MJ, Harmer CJ, Flint J, Cowen PJ, Goodwin GM (2005). Enhanced early morning salivary cortisol in neuroticism. American Journal of Psychiatry 162, 807-809.

Pruessner J, Wolf O, Hellhammer D, Buske-Kirschaum A, von-Auer K, Jobst S, Kaspers F, Kirschbaum C (1997). Free cortisol levels after wakening: a reliable biological marker for the assessment of adrenocortical activity. Life Sciences 61, 2539-2549.

Ribiero SC, Tandon R, Grunhaus S, Greden JF (1993).

The DST as a predictor of outcome in depression: a meta-analysis. American Journal of Psychiatry 150, 1618-1629.

Wust S, Federenko I, Hellhammer D, Kirschbaum C (2000). Genetic factors, perceived chronic stress and the free cortisol response to awakening. Psychoneuroendocrinology $25,707-720$. 